\title{
El problema de
}

la universidad

en Venezuela y

los lugares para

su resistencia

(im)posible

en tiempos de

postautonomía

Eleonora Croquer Pedrón

Recebido em: 30 de abril de 2018

Aceito em: 25 de maio de 2018
Doutora em Filologia Hispano-Americana

(2004, Universitat de Valencia,

España), mestre em Literatura Latino-

Americana (1995, Universidad Simón

Bolívar, Venezuela) e bacharela em

Letras (1991, Universidad Central

de Venezuela). É diretora do Instituto

de Altos Estudios de América Latina e

responsável pelo Centro de Investigaciones

Críticas y Socioculturales da Universidad

Simón Bolívar (Caracas-Venezuela), onde

trabalha como professora e pesquisadora

desde 1992. Publicou os livros El

sesto de Antísona o la escritura como

responsabilidad (Clarice Lispector, Diamela

Eltit y Carmen Boullsa) (Santiago de

Chile: Cuarto Propio, 1999) e Escrito

con rouge. Delmira Agustini (1886-

1914), artefacto cultural (Rosario: Beatriz

Viterbo, 2009). Ela conta, aliás, com

mais de 40 publicações entre capítulos

de livro e artigos especializados. Suas

linhas de pesquisa são: literatura escrita por mulheres na América Latina, problemas de crítica cultural, imagem e cultura visual nos entre-séculos XIX e XX latino-americanos, autorias excêntricas da literatura e $\mathrm{O}$ arte. Contato: eleonora.croquer.pedron@ smail.com 


\section{PALABRAS CLAVE:}

Universidad; Autonomía;

Resistencia; Institutos; Centros y grupos de investigación.
KEYWORDS: University;

Autonomy; Resistance; Institutes;

Centers and research groups.
El presente ensayo en torno al problema de la universidad, situado en las circunstancias que atañen a las universidades autónomas en la Venezuela contemporánea, se propone como una llamada de atención a la urgencia de asumir una reflexión sobre la universidad no sólo tendiente a revisar lo que ha sido la tradición en la que se inscribe, sino también a postular las condiciones imprescindibles para su supervivencia en tiempos de postautonomía como los que parecen imponerse en el presente mundializado. En este orden de ideas, fija su atención en los institutos, centros y grupos de investigación como lugares capaces de hacer posible una resistencia que parecería imposible, toda vez que ellos cuentan con las condiciones necesarias para la generación de una comunidad de pensamiento abierta a la proposición de nuevas perspectivas de investigación y estudio, al tiempo que permiten una integración distinta de docentes y estudiantes.

The present essay about the problem of the university, located in the circumstances that concern the autonomous universities in contemporary Venezuela, is proposed as a wake-up call to the urgency of assuming a reflection on the university tending not only to review what has been the tradition in which it is registered, but to postulate the essential conditions for its survival in times of post-autonomy as those that seem to be imposed in the slobalized present. In this order of ideas, it fixes its attention on the institutes, centers and research groups as places capable of making possible a resistance that would seem impossible, since they have the necessary conditions for the generation of a community of thought open to Proposal of new perspectives of research and study, while allowing a different integration of teachers and students. 


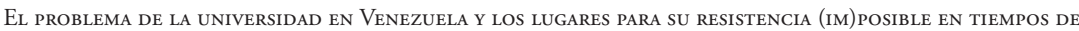
POSTAUTONOMÍA

\section{Pensar (en) LA UnIVERSIDAD: RESPONSABILIDAD Y SUPERVIVENCIA}

[E]s en las situaciones más crepusculares, más occidentales de la Universidad occidental en donde se multiplican las oportunidades de este twinkling del pensamiento. En periodo de "crisis", como suele decirse, de decadencia o de renovación, cuando la institución está ontheblink, la provocación que es preciso pensar reúne en el mismo momento el deseo de memoria y la exposición de un porvenir, la fidelidad de un guardián lo suficientemente fiel como para querer conservar incluso la oportunidad del porvenir, dicho de otra forma la singular responsabilidad de lo que aún no tiene y aún no está.

(Jacques Derrida. "Las pupilas de la Universidad. El principio de razón y la idea de la Universidad")

Ante todo, quiero hacer énfasis en lo real de una coyuntura terrible que ninguna reflexión seria sobre la universidad, planteada desde Venezuela y en el ahora responsable de su enunciación, podría eludir: las universidades autónomas en el país son hoy el resto de un progresivo y sistemático proceso de arruinamiento económico y académico que, más allá de las contradicciones que hicieran al estado de crisis de sus cimientos en Occidente hacia la segunda mitad del siglo xx (Readings, 1996), pasa por la devaluación del trabajo que en ellas se realiza y por el desconocimiento de sus necesidades presupuestarias básicas, por parte del Estado del cual depende su manutención. Un Estado que en otros momentos de su historia las había sostenido materialmente, aunque nunca hubiera mantenido con 
ellas una relación poco conflictiva, como bien expone Alexis Márquez Rodríguez (2005); pero que a lo largo de estas últimas dos décadas parece haberlas arrojado a las derivas inciertas de la precariedad.

Tanto la devaluación del trabajo a la que me refiero, como el empobrecimiento que la reducción presupuestaria va generando a su paso, son comunes a los que ocurren en todos los órdenes de la vida profesional del país, y asimismo cónsonos con la precariedad de una existencia humana desnudada de todas sus garantías, por las razones económicas, políticas y morales que hacen al quiebre radicalizado de la Nación en el marco del desmantelamiento a que ha sido gradualmente sometida en nombre del supuesto "proyecto bolivariano" que rige los últimos casi veinte años de gobierno "revolucionario". Pero además, en el caso de las universidades autónomas, abiertamente opositoras al proceso de reconfiguración del escenario nacional que ha ido teniendo lugar en este último periodo de su historia, tales devaluación y empobrecimiento adquieren conotaciones retaliativas evidentes, que se suman a otras acciones excedidas del Estado en cuanto a su intervención en decisiones académicas, administrativasy gremiales de la institución que lejos están de resolver lo que podrían considerarse como sus problemas estructurales. En este contexto, sin duda, es el estatuto de universidad autónoma, en tanto pública y autogestionada, el que está en situación de riesgo en el país; toda vez que el aparato gubernamental desconoce tanto sus necesidades económicas como la legitimidad de su autonomía, al tiempo que la respuesta opositora inmediata y desesperada apunta más bien a señalar que la privatización de estas universidades - o la 
El problema de LA UNIVERSIDAd EN VENEZUELA Y LOS LUGARES PARA SU RESISTENCIA (IM) POSIBLE EN TIEMPOS DE POSTAUTONOMÍA

búsqueda de un amparo alternativo en el vínculo con la empresa privada podría ser una "solución" al estado insostenible de su sojuzgamiento.

Entre tanto, en el entre tanto de un arruinamiento precipitado de manera salvaje por la pendiente inflacionaria que ocupa la vida de los venezolanos con cada vez mayor contundencia, la desprofesionalización y la diáspora se imponen, ante los ojos indiferentes del cuerpo social respecto del estado agónico que aqueja a estas instituciones y que a nadie parece importarle demasiado. A lo largo de estas décadas y con cada vez mayor intensidad, un gran porcentaje de profesores va abandonando las aulas de estas universidades para migrar hacia otros espacios laborales que le permitan hacer frente a sus circunstancias, dentro y fuera del país; y los que permanecemos nos vemos obligados a asumir otros trabajos adicionales al que la universidad compromete en detrimento sobre todo de las labores de investigación y extensión que, tan importantes como la docencia, harían a un desarrollo profesional pleno del profesor universitario y de la universidad que certifica el carácter especializado de las prácticas que le corresponden. Esta dramática situación tiende a recrudecerse con el importante abandono de las universidades autónomas por parte de un estudiantado que también migra: hacia otras universidades privadas del país, que le garantizan una mínima estabilidad operativa para el desarrollo de sus estudios, y/o hacia otros países, en aras de una promesa de bienestar que, como bien sabemos, no siempre se realiza...

No es de esto, sin embargo, de lo que hablaré en mi presente reflexión, aun cuando no deje de insistir en que otro sería probablemente el escenario si tanto el presupuesto universitario como los sueldos de los profesores que 
en ella habrían de ejercer su profesión representaran alguna garantía de sustento. Y/o, también, que otro sería el escenario si esta universidad no tuviera que confrontarse con el presente desesperanzado de un país que en gran medida se presenta ante las gentes como "sin otra salida posible que el éxodo”. Hablaré, más bien, de la universidad venezolana en el tiempo de postautonomía que parece dominar el orden mundializado en el cual no deja de inscribirse la singularidad de su autonomía sitiada, haciendo uso del término propuesto por Josefina Ludmer para aproximarse a una comprensión del presente, en lo que tiene de absorción de las formas modernas de administración del poder de entre las cuales despuntara otrora la idea de autonomía, en la instancia de otros poderes omniabarcantes y voraces que pocos espacios dejan para una existencia soberana e incondicionada como la que pulsa en el origen de las universidades autónomas. Hablaré, asimismo, de las universidades autónomas sitiadas en la Venezuela contemporánea, a través de lo que considero la expresión sintomática de la inadecuación de la universidad a un presente que parece negarle tanto un lugar como un sentido a la "razón de ser" sobre la cual se asienta su existencia como fundamento y como fundación, a partir de la discusión desplegada por Jacques Derrida, en defensa de una responsabilidad subjetivamente asumida por el pensamiento al interior y más allá de sus muros, tanto con la memoria de su fundación como con la imprescindible revisión de sus fundamentos de cara al futuro. Y hablaré, finalmente, de los institutos, centros y grupos de investigación; lugares de resistencia que podrían restituirle un sentido a su supervivencia en la emergencia de nuevas comunidades de pensamiento capaces no sólo de revisar críticamente una tradición de la cual serían herederos últimos, sino 
El problema de la universidad EN Venezuela y loS LUGARES PARA SU RESISTENCIA (IM)POSIBLE EN TIEMPOS DE POSTAUTONOMÍA

de dejarse interpelar de otra manera por lo contemporáneo. Es decir, por eso de lo contemporáneo que aún podría suscitar una respuesta distinta de la universidad a la mera aceptación del mandato inclemente de los poderes que lo organizan.

Porque es, entre otras cosas, un vacío de sentido lo que se materializa allí donde la desprofesionalización y la diáspora se imponen como evidencia del arruinamiento de una universidad que ya no sirve a los fines ideológicos del Estado desmantelado, y que, en su pobreza manifiesta, no termina de satisfacer las exigencias de un mercado cada vez más descarnado y competitivo. Un vacío de sentido que, más allá de lo inmediato - la pauperización de las condiciones materiales del trabajo -, refiere también a un debilitamiento - histórico, epocal - de la "razón de ser" que una vez hizo causa de deseo en el profesor que emprendía su vida en función de un cierto compromiso con la "verdad" que la universidad representaba, y que en gran medida sostuvo los diversos debates y controversias que animaron cada una de sus últimas revisiones a lo largo del siglo xx. Y un vacío de sentido que, en definitiva, nos confronta a todos los que aún insistimos en permanecer con la imperiosa necesidad de reinventar los términos de nuestra supervivencia; es decir, de hacer de la resistencia en apariencia imposible, una posibilidad de resistencia abierta a lo porvenir... Porque es en nombre del porvenir de la universidad - esa universidad no toda y no del todo necesariamente reducida a ser la corporación burocrática que en el presente parece imponerse como horizonte inmediato de subsistencia - que el pensamiento sobre su fundación y sus fundamentos puede permitirnos aún reconciliarnos con la 
idea de seguir realizando lo que estamos llamados a realizar desde el tiempo largo de una historia que no puede y no debe haber sucedido en vano.

“Existe hoy en día, en lo que respecta a la universidad, lo que se llama una 'razón de ser'?", se interroga Derrida en el texto que dedica a "Las pupilas de la Universidad. El principio de razón y la idea de la Universidad" (1997, p.117). Y apunta a continuación: “Tener una 'razón de ser' es tener una justificación para existir, tener un sentido, una finalidad, una destinación. Es asimismo tener una causa, dejarse explicar, según el 'principio de razón', por una razón que es también una causa (ground, Grund), es decir también un fundamento y una fundación" (p.117). Dicho esto y problematizado, más adelante en su argumentación, el filósofo vuelve a preguntarse "¿Cuál es, pues, mi intención? ¿Qué perseguía al presentar las cosas de este modo?” (p.131). A lo cual responde: "Pensaba, sobre todo, en la necesidad de despertar o de volver a situar una responsabilidad en la Universidad o ante la Universidad, y ello se forme o no parte de la misma” (p.131). Y continúa:

De esa nueva responsabilidad a la que me refiero sólo puede hablarse apelando a ella. Se trataría de la de una comunidad de pensamiento para la cual la frontera entre investigación fundamental e investigación finalizada no resultase ya segura, al menos no en las mismas condiciones que antes. La denomino comunidad de pensamiento en sentido lato (at large) mejor que de investigación, de ciencia o de filosofía ya que dichos valores están muy a menudo sometidos a la autoridad no-cuestionada del principio de razón 


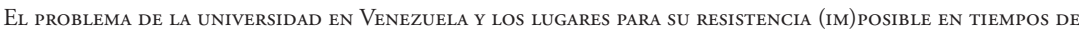
POSTAUTONOMÍA

[...]. Una comunidad semejante se cuestiona sobre la esencia de la razón y del principio de razón, sobre los valores de fundamental, de principial, de radicalidad, de la arkhè en general, e intenta sacar todas las consecuencias posibles de dicho cuestionamiento. Un pensamiento semejante no es seguro que pueda agrupar a una comunidad o fundar una institución en el sentido tradicional de estas palabras. Ha de re-pensar también aquello que se denomina comunidad e institución. Debe descubrir asimismo, tarea infinita, todas las astucias de la razón finalizante, los trayectos por medio de los cuales una investigación aparentemente desinteresada puede ser indirectamente reapropiada, empleada de nuevo por programas de todo tipo (p.133; énfasis del autor).

Para Derrida, "[v]olver a situar una responsabilidad en la Universidad”, supondría fundamentalmente volver a pensar en la universidad repensando la universidad, con miras "a transformar de forma consecuente los modos de escritura, la escena pedagógica, los procedimientos de co-locución, la relación con las lenguas, con las demás disciplinas, con la institución en general, con su fuera y su dentro" (p.134). Y, en este orden de ideas, la fidelidad a la que aspira su razonamiento, una fidelidad capaz de velar tanto por la memoria de un pacto cifrado en el pasado como por la reflexión concentrada en proteger lo que aún no ha sido, actuaría no ya en nombre de un principio de razón cuestionado, cuanto en función de la emergencia de una nueva comunidad de pensamiento surgida de ese cuestionamiento 
y comprometida con la supervivencia de la universidad y de lo que en ella podría volver a resplandecer como sentido...

Más allá de las distancias que refieren el pensamiento de Derrida a la universidad en otros contextos y atravesada por problemáticas distintas, podríamos no obstante considerar que ante la autonomía sitiada en las universidades venezolanas, y en tiempos de postautonomía como los que se imponen hoy en el Occidente mundializado, habría que volver a pensar (en) la universidad. Esto es: asumir la responsabilidad de pensar en el parpadeo que se abre con el recrudecimiento de su crisis - "crisis no moderna de la universidad moderna", como explica Willy Thaller (1996) en el libro homónimo que dedica a problematizar las circunstancias de cambio con las cuales debe confrontarse hoy una institución heredera de otros tiempos - y de pensarla, a la vez, respecto de la historia de su fundación y del porvenir de su supervivencia. En ese doble acto de pensamiento responsablemente asumido, y en lugar de entregarse a una carrera ciega por emular los cambios que el presente parece exigirle a la institución, algo así como una autonomía universitaria anacrónicamente revestida de un nuevo sentido - o sea, una autonomía que se postule en defensa de la existencia incondicionada de la universidad y del pensamiento que se despliega dentro y fuera de sus aulas - puede volver a iluminar su destino; en vista de que, como afirma asimismo Jacques Derrida en otra comunicación desarrollada al respecto, es esa existencia incondicionada la que podría aún justificar su resistencia (im) posible hacia el futuro. Una resistencia frente ala cual deberían volver a dirimirse tanto la función social y política de la institución, como la fe 


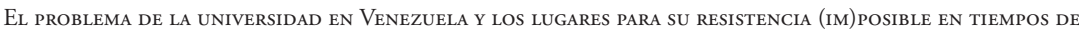
POSTAUTONOMÍA

que profesa cada uno de los profesores que aún insisten en hacer de la vida académica en el campus una "profesión de fe":

[L]a universidad moderna debería ser sin condición. Entendamos por "universidad moderna" aquella cuyo modelo europeo, tras una rica y compleja historia medieval, se ha tornado predominante, es decir "clásico", desde hace dos siglos, en unos Estados de tipo democrático. Dicha universidad exige y se le debería reconocer en principio, además de lo que se denomina la libertad académica, una libertad incondicional de cuestionamiento y de proposición, e incluso, más aún si cabe, el derecho de decir públicamente todo lo que exigen una investigación, un saber y un pensamiento de la verdad [...]

Esta universidad sin condición no existe, de hecho, como demasiado bien sabemos. Pero, en principio y de acuerdo con su vocación declarada, en virtud de su esencia profesada, ésta debería seguir siendo un lugar de resistencia crítica - y más que crítica - frente a todos los poderes de apropiación dogmáticos e injustos $[\ldots]$

Consecuencia de esta tesis: al ser incondicional, semejante resistencia podría oponer la Universidad a un gran número de poderes: a los poderes estatales (y, por consiguiente, a los poderes políticos del Estado-nación así como a su fantasma de soberanía indivisible: por lo que la universidad sería de antema- 
no no sólo cosmopolítica, sino universal, extendiéndose de esta forma más allá de la ciudadanía mundial y del Estado-nación en general), a los poderes económicos (a las concentraciones de capitales nacionales e internacionales), a los poderes mediáticos, ideológicos, religiosos y culturales, etc., en suma, a todos los poderes que limitan la democracia por venir (Derrida, 1988).

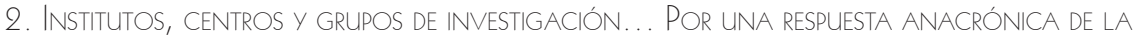

\section{UNIVERSIDAD SITIADA}

[C]ualquiera sean sus razones, para mí la postautonomía es un modo de pensar el cambio: en el lugar del autor, en los modos de leer, en el régimen de realidad y en el régimen de sentido. Para poder hacer activismo cultural necesito pensar ese otro régimen de realidad, ese otro lenguaje y ese otro sentido.

(Josefina Ludmer "Literaturas postautónomas: otro estado de la escritura”)

A fin de pensar lo que identifica como un cambio en la noción de literatura, y de cara a cierta posibilidad de seguir asumiendo la crítica como una forma de activismo cultural - es decir, de resistencia contra "todos los poderes que limitan la democracia por venir" -, la investigadora argentina Josefina Ludmer se refiere a un nuevo orden postautonómico mundializado que, ante la evidencia de una autonomía de la escritura literaria desplazada, la confronta con la necesidad de diseñar categorías también distintas de 


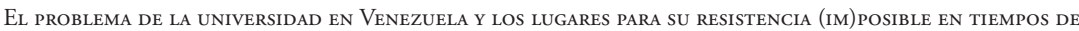
POSTAUTONOMÍA

aproximación a lo que ya no puede ser comprendido como una "esfera separada de otras esferas o prácticas":

Para caracterizar la literatura de hoy uso el concepto de postautonomía, y para periodizarla uso "lo que viene después". En los dos casos rige la idea de lo post. Usar la palabra post implica que las divisiones no son tajantes ni proceden tan dialécticamente. La característica de lo que viene después es que no es anti ni contra sino alter, que no hay un corte total con lo anterior, que el pasado está presente en el presente y persiste junto con los cambios.

Uso las dos constelaciones (autonomía y postautonomía) y algunos instrumentos conceptuales (como "en sincro" y "en fusión") para pensar el presente. No pretendo que las constelaciones y los instrumentos conceptuales sean verdaderos ni falsos. Son solo instrumentos conceptuales que sirven de base de alguna especulación y pueden ser importantes para el activismo cultural.

La postautonomía sería la condición de la literatura en la actualidad: el modo en que se podría imaginar el objeto literario, y también la institución literaria. Uso la idea, la palabra si se quiere, de postautonomía para marcar que lo central es la relación con la autonomía, con el pasado en el presente. La tensión y la oscilación entre postautonomía y autonomía definiría este presente. 
Decir que estamos en la era de la postautonomía significa reconocer que han cambiado, en los últimos 20 años por lo menos, los modos de leer y los modos de producción del libro. Que ha cambiado el objeto literario. Que ya no me sirve solamente la idea de autonomía, donde la literatura es pensada o imaginada como esfera separada y diferente de otras esferas o prácticas (sobre todo separada de lo político). ${ }^{1}$

Dos ideas nos parecen particularmente relevantes de la reflexión propuesta por Ludmer acerca del campo específico de la literatura, así como de los lugares ocupados por el autor, los modos distintos de leer y los regímenes de realidad y de sentido hoy cambiantes a los que remite. Por una parte, la identificación de un desplazamiento que, en el presente, hace confusa la existencia autónoma de prácticas que, como la literatura, en otros momentos de la historia de la cultura gozaron de algún tipo de incondicionalidad respecto de los poderes políticos y económicos que, sin embargo, promovieron y sostuvieron su realización. Y, por otra, la certeza de que sólo mediante una comprensión de la situación de cambio del presente es posible diseñar una acción (crítica) de resistencia... Porque, de nuevo, es el diseño de una posibilidad de resistencia a lo que apunta en definitiva la reflexión que propongo a propósito de la universidad en Venezuela, aunque ello suponga una apuesta anacrónica evidente en defensa de la autonomía universitaria como instancia de sentido a la cual apelar en el tiempo de postautonomía - histórico, epocal - en el cual se inscribe su autonomía

1 En: http://www.revistadossier.cl/literaturas-postautonomas-otro-estado-de-la-escritura/. 
El problema de la uniVersidad EN VeNEZUELA y loS LUGARES PARA SU RESISTENCIA (IM)POSIBLE EN TIEMPOS DE POSTAUTONOMÍA

sitiada -esto es: amenazada, tanto por el asedio gubernamental, como por las nuevas formas de dependencia económica que la someten a las demandas de un mercado que no necesariamente y no siempre reconoce el valor de las tradiciones, debates y pugnas que pulsan al interior de los saberes articulados en Occidente más allá de su instrumentalización burocrática.

En este sentido, la observación de Ludmer nos permite un acercamiento a la comprensión del estado de postautonomía universitaria que parece suceder de diversas maneras a las diferentes manifestaciones de la crisis de la universidad manifiesta en Occidente durante la segunda mitad del siglo xx"crisis no moderna de la universidad moderna" (Thayer, 1996). O sea: un acercamiento a partir de lo que podría ser concebido como un cambio en la noción de universidad y que, como afirman Raúl Rodríguez Freire y Andrés Maximiliano Tello, no sólo ha diluido "[1]as murallas reales y ficticias que otrora delimitaron el espacio universitario, que le otorgaron su soberanía" (2012, p.17), sino que la ha arrojado a esa suerte de "descampado" en el que parece dirimirse su cotidianidad:

Por mucho tiempo la afirmación de la autonomía universitaria sirvió como garante de una contienda en donde la facultad inferior, el pensamiento crítico o el trabajo intelectual se encargaban de poner en cuestión el saber instituido y utilitario con sus doctrinas, que funcionaban en el resto del cuerpo social. Pero las murallas de la universidad fueron desbordadas no sólo por la expansión de sus conocimientos, sino por el propio principio liberal que propiciaba su campo de acción autónomo, su laizzes-faire. Así, en el capita- 
lismo contemporáneo la coincidencia entre los momentos de producción y circulación -de cualquier mercancía, incluyendo al conocimiento mismo, y a quienes portan o encarnan dicho conocimiento- se traduce también en la coincidencia entre universidad y mercado -y también entre mercado y democracia. Como efecto de esta convergencia se puede entender el mercado global de la educación surgido en las últimas décadas. Según esta nueva condición desterritorializada, no sólo la labor de investigación, formación y producción, así como la circulación de conocimientos universitarios, se rige por el mercado, sino que además la propia administración de las instituciones de educación superior se realiza desde una lógica empresarial, reterritorializando a la universidad en nuevos procedimientos corporativos supeditados al mundo de los negocios, del managament.

La libertad de circulación de la cual goza hoy la universidad flexible, para diseminarse en sedes nacionales e internacionales, fragmentarse en programas de investigación y en ofertas curriculares, presenciales y virtuales, se traduce paradójicamente en la hipoteca de su autonomía, tal como ésta fue pensada modernamente $[\ldots]$. Las murallas reales y ficticias que otrora delimitaron el espacio universitario, que le otorgaron su soberanía, se han diluido, de manera que ya no existe un campo de acción autónomo para la universidad, ni menos un "campo cultural" que la cobije; habitamos un descampado -un terreno baldío, parafraseando a T. S. Eliot- que la universidad contribuye, y no poco, a mantener. Una universidad líquida, en la terminología de Zygmunt Bauman (Rodríguez Freire y Tello, 2012, p.16-17; énfasis de los autores). 


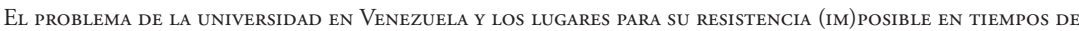
POSTAUTONOMÍA

Al respecto, también Willy Thayer expone con suficientes argumentos hasta dónde la Universidad moderna que se postula en torno a la idea de autonomía del pensamiento pierde sentido en un mundo que se reorganiza como diseminación del conocimiento en las nuevas redes de difusión digital de la información y hegemonía de la opinión pública sobre la reflexión especializada que el claustro universitario garantizaría. El debilitamiento del Estado-nación característico de los nuevos rumbos que asume la política en el marco de la economía transnacional, por otra parte, implica tanto el borramiento de la función rectora de la universidad en la formación de cierto modelo de ciudadanía que en otros momentos de su historia justificaba plenamente su papel formativo respecto del estudiantado, como el devenir puramente instrumental de sus funciones en la sociedad:

Los vientos que corren hoy en día respecto del saber, el Estado, el pueblo, la lengua, el espíritu, la verdad, la naturaleza, la historia, el género humano, si bien no son contrarios a la "industria universitaria" (Kant) ni a la circulación ampliada del saber en el mercado, ni al imperativo contextual de profesionalización, han moderado el brillo, el fulgor epopéyico con que la investía su papel de heroína de la historia, conductora de naciones y de la humanidad en su conjunto.

Todos los emblemas de su épica constituyen ahora su kitsch, el kitsch de la universidad, el kitsch del saber y de la historia. Y no podía sino ser así en un contexto donde lo heroico de la ciencia y de la creación, ligado naturalmente 
al dramatismo teleológico y la trascendencia utópica, ha sido paulatinamente relevado por la inmanencia operacional de las dietas curriculares y los circuitos metódicos de apropiación por cuotas de inteligencia profesional, las mallas curriculares de acreditación transitables con la liberalidad de un supermercado o de una mesa sueca, donde a partir de una carta de especialidades y códigos en oferta, y a condición de disponerse en los hábitos en curso, cada cual puede organizar su propio refrito técnico-profesional. Los emblemas e impulsos humanistas y progresistas de la universidad moderna - emblemas que pueblan indiscriminadamente los discursos rectorales en la actualidad - no tienen más función que la de adornar y recubrir las relaciones público/publicitarias de la universidad. Contrariamente, es la administración universitaria la que acota y opera el efecto y alcance de su significación.

No se habita ya la universidad en el sentido y grosor temporal que antaño generaban tales emblemas y promesas. La corrosión del principio especulativo (unidad interna y orgánica del saber); así como la inmanentización del principio práctico teleológico del progreso, principios que acompañaban legitimando y dando un sentido meta-operacional a las prácticas de saber (investigación, docencia) habrían sido relevados por la performance del funcionamiento y la operatividad tecnológica; y por la mera contigüidad comunicativa de los saberes y las prácticas. Con tal deslegitimación - o legitimación sólo por la performance del funcionamiento - la universidad no estaría destinada a formar una élite capaz de guiar a la nación en su emancipación, o profesionalizar las fuerzas de trabajo en la teleología. Educación > 
El PROBlema de la UNIVERSIDAd EN VENEZUELA y los LUGARES PARA SU RESISTENCIA (IM) POSIBLE EN TIEMPOS DE POSTAUTONOMÍA

producción > ganancia > libertad > felicidad; sino parece más bien destinada a proporcionar agentes para ocupar los puestos pragmáticos de los que las empresas tienen necesidad. La universidad no proyecta la universitario más allá de la traducción de la rutina curricular en rutina profesional, la manipulación acertada y eficaz, el proceso autorreferido del trabajo técnico y la reproducción de un "sí mismo" mejorado en el standing; no otorga futuro, aparte de las fechas límite del endeudamiento crediticio; y no abre expectativas mayores a las de la adquisición de códigos, claves y cosméticas de ingreso y egreso en el mercado absoluto (Thayer,1996, p.44-46).

¿Desde dónde, entonces, aproximarnos hoy a una reflexión acerca de la universidad en Venezuela que, sin desconocer la dramática coyuntura que la azota, sea al mismo tiempo capaz de dar cuenta de los cambios (históricos, epocales) en los cuales no deja de inscribirse su autonomía sitiada? ¿Y desde dónde, en consecuencia, volver a encontrar un sentido que justifique su supervivencia como espacio de resistencia crítica a los "poderes que limitan la democracia por venir" en el país y en Occidente? Sin duda, pensar (en) la Universidad venezolana y en su supervivencia posible es pensar más allá de su adecuación acrítica y cómplice a un comportamiento burocrático como el que espera de ella la reconfiguración mundial de los poderes y de sus nuevas maneras de sojuzgamiento; y es, asimismo, volver a otorgarle una responsabilidad política que permita y promueva la emergencia de nuevas comunidades de pensamiento capaces de proponer respuestas críticas respecto de un futuro aún posible para la construcción de un destino 
distinto en el país. Quizá esa reflexión podría no sólo hacerle frente a la "crisis no moderna de la universidad moderna", sino encontrar los términos para referirse a las nuevas potencias que pulsan en espera de su emergencia tras las formas gastadas de su impotencia actual. En su texto sobre "La universidad sin condición", Jacques Derrida destaca como rasgo de esa potencia la posibilidad de generar un pensamiento capaz de confrontarse con la "verdad" que haría a la profesión de fe propia de la universidad en términos no sólo interrogativos, sino "afirmativos" y "performativos". Esto es, un pensamiento capaz de producir acontecimientos: "de hacer que algo le ocurriese, sin necesariamente traicionarlo, a ese concepto de verdad o de humanidad que conforma los estatutos y la profesión de fe de toda universidad" (1998).

En el marco de esta reflexión, y al margen de la reconfiguración de la universidad como corporación burocrática de una investigación y una docencia puestas al servicio de los diversos poderes que hoy organizan lo social, una reconfiguración que en Venezuela se ve a cada paso desplazada por los problemas específicos a los cuales debe día a día hacerle frente, los institutos, centros y grupos de investigación pueden ser espacios capaces de explorar esa potencia performativa del pensamiento con miras a la generación de nuevas posibilidades de sentido para su supervivencia. Ello si concebidos como lugares de encuentro entre investigadores, profesores y estudiantes, reunidos en torno al interés común por la elaboración conceptual inquieta; y a lo que ésta supondría del diseño de nuevas preguntas, objetos de estudio y acciones de cambio dentro y fuera de la Universidad. Situados satelitalmente respecto de la estructura universitaria, de la cual son suplementarios, los 
El problema de la universidad EN Venezuela y loS LUGARES PARA SU RESISTENCIA (IM)POSIBLE EN TIEMPOS DE POSTAUTONOMÍA

institutos, centros y grupos de investigación permiten y promueven una textura interdisciplinaria para el abordaje de interrogantes transversales a las que impone la capacitación técnica del estudiantado; y, en consecuencia, la apertura de la investigación y el estudio hacia otro tipo de mirada susceptible de ser interpelada por una contemporaneidad que en mucho excede los marcos disciplinares del saber. Permiten y promueven, además, un tipo de acción que, cuando asentada sobre la cualidad heteronómica de su libre albedrío, sirve a la promoción de nuevas alternativas de agenciamiento tendentes a fortalecer tanto los vínculos entre los diversos actores que integran la comunidad universitaria como esos otros que deben necesariamente producirse entre la universidad y lo social.

Acerca de esta última afirmación, y a manera de ejemplo, quiero referirme al caso del Instituto de Altos Estudios de América Latina de la Universidad Simón Bolívar de Venezuela; y, más precisamente, a la experiencia de investigación y formación de estudiantes de cuarto nivel "Formas profanas, laboratorio de crítica cultural", del Centro de Investigaciones Críticas y Socioculturales, uno de los cinco centros que se alojan en su estructura. En torno a una mesa de debate en la cual se cruzaron lecturas teóricas y revisiones de algunas posiciones excéntricas de la crítica cultural en América Latina, tal como se gestaron más allá del latinoamericanismo de los años setenta y de los debates hegemónicos de los Estudios Culturales en la academia norteamericana, "Formas profanas" se concentró en estimular la singularidad de cada una de las investigaciones que los estudiantes que participaron fueron diseñando a lo largo de dos años de encuentros. El Centro de Investigaciones Críticas y Socioculturales, formado por profesores de diversos departamentos de la universidad y abierto 
a la integración de disciplinas heterogéneas del campo de las humanidades, promovió los encuentros y los canalizó hacia la participación en congresos y publicaciones de la especialidad. Su modelo fue heterológico; y su punto de partida, una interrogación acerca del estado actual de la crítica cultural. Cada participante desarrolló la singularidad de su propuesta de investigación en un entorno de pensamiento y de respeto a la diferencia que, a la manera de un laboratorio, estimuló el libre albedrío y la responsabilidad para con las lecturas discutidas, la propia investigación y su lugar en la universidad. Una experiencia de ese tipo no hubiera sido posible en otro espacio, constreñido por las exigencias burocráticas de una investigación y un pensamiento puesto al servicio de la mera adquisición de un título. Y no hubiera sido posible, tampoco, fuera del espíritu de trabajo conjunto que, libre de la pretensión de fundar una institución, se nutrió de la convicción conjunta de estar realizando un trabajo de pensamiento cifrado en el común del encuentro.

Así, "Formas profanas" se consolidó como un espacio de enunciación académica experimental que se desplazaba problemáticamente entre la prestidigitación positivista y el "todo vale" postmoderno bajo el cual parece hoy dirimirse el campo de los saberes sociales y humanos. Se trataba, por ello, de la apuesta de un grupo de estudio y acción conceptual gestado entre investigadores consolidados y en formación, en nombre de la articulación de nuevas constelaciones teóricas capaces de sostener prácticas también diversas de aproximación al estudio de las formas, imágenes y discursos de la cultura, así como de la tensión nunca del todo resuelta que ellas traducen con lo Real de los acontecimientos históricos, sociales, subjetivos y libidinales que 
El problema de la universidad EN Venezuela y loS LUGARES PARA SU RESISTENCIA (IM)POSIBLE EN TIEMPOS DE POSTAUTONOMÍA

impulsan los devenires de su materialidad. Y todo ello sin instrumentalizar ni al sujeto investigador ni al objeto de su investigación.

La experiencia "Formas profanas" sesostuvo durante dos ańos deencuentros que se realizaban una vez a la semana; y, más allá de las conferencias y artículos que se desarrollaron en su seno, produjo sobre todo una conciencia de la responsabilidad que supone asumir un objeto de estudio e investigación en la trayectoria intelectual de un sujeto capaz de responder performativamente a las condiciones que lo adversan. El Centro de Investigaciones Críticas y Socioculturales, marginal y suplementario respecto del curso emprendido por la Universidad Simón Bolívar hacia las transformaciones que la época parece imponerle, y más allá de la precariedad de sus circunstancias, logró abrir espacios para la emergencia de una comunidad de pensamiento que hoy se mantiene viva en medio de la desesperanza y el arruinamiento.

\section{REFERENCIAS BIBLIOGRÁFICAS}

Derrida, Jacques. "Las pupilas de la Universidad. El principio de razón y la idea de Universidad”. En: Cómo no hablar y otros textos. Barcelona: Proyecto a ediciones, 1997, pp. 117-138.

(1998). "La universidad sin condición". Recuperada en línea <https://rafaelfdiazv.files.wordpress.com/2009/06/derrida-jacques-universidad-sin-condicion 1.pdf>.

Ludmer, Josefina. "Literaturas postautónomas: otro estado de la escritura”. Disponible en: <http://www.revistadossier.cl/literaturas-postautonomas-otro-estadode-la-escritura/ >.

Márquez Rodríguez, Alexis. Autonomía universitaria y revolución. Caracas: Equinoccio, 2005. 
Readings, Bill. The University in Ruins. Cambridge: Harvard UP, 2006.

Rodríguez Freire, Raúl y Andrés Maximiliano Tello. "La universidad en ruinas. A modo de presentación”. En Raúl Rodríguez Freire y Andrés Maximiliiano Tello (eds.). Descampado. Ensayos sobre las contiendas universitarias. Santiago de Chile: Sangría editora, 2012.

Thayer, Willy. La crisis no moderna de la universidad moderna (epilogo de El conflicto de las facultades). Santiago de Chile: Cuarto propio, 1996. 